\title{
Surgical Anatomy of the Medial Sural Artery Perforator Flap
}

\author{
Man-Zhi Wong, M.B.B.S., M.R.C.S., M.Med. ${ }^{1}$ Chin-Ho Wong, M.B.B.S., M.R.C.S., M.Med., F.A.M.S. ${ }^{2}$ \\ Bien-Keem Tan, M.B.B.S., F.R.C.S., F.A.M.S. ${ }^{1}$ Khong-Yik Chew, M.B.B.S., M.R.C.S., M.Med. ${ }^{1}$ \\ Shian-Chao Tay, M.B.B.S., F.R.C.S., F.A.M.S., F.I.C.S. ${ }^{3}$
}

${ }^{1}$ Department of Plastic, Reconstructive and Aesthetic Surgery,

Singapore General Hospital, Singapore

2 W Aesthetic Plastic Surgery

${ }^{3}$ Department of Hand Surgery, Singapore General Hospital, Singapore

\begin{abstract}
Address for correspondence and reprint requests Chin-Ho Wong, M.B.B.S., M.R.C.S., M.Med, F.A.M.S., W Aesthetic Plastic Surgery, \#08-42 Mount Elizabeth Novena Specialist Center, 38 Irrawaddy Road, Singapore 329563 (e-mail: wchinho@hotmail.com).
\end{abstract}

J Reconstr Microsurg 2012;28:555-560.

\begin{abstract}
Background The medial sural (medial gastrocnemius) perforator flap is a thin flap with a long pedicle. It has tremendous potential for applications in a variety of soft-tissue defects. We aimed to further clarify the vascular anatomy of the medial sural region and establish a safe approach for elevation of this flap.

Methods Ten fresh cadaveric lower limbs were injected and used in this study. We identified the locations and courses of the medial sural artery perforators and correlated them to anatomic landmarks.

Results The medial sural artery divides into two branches, a medial and lateral branch. Correspondingly, musculocutaneous perforators supplying the overlying skin were oriented in two parallel vertical rows, along the course of the lateral or medial branch of the medial sural artery. Two to six perforators were located $6 \mathrm{~cm}$ to $22.5 \mathrm{~cm}$ from the popliteal crease. Perforators from the lateral row, nearer the posterior midline, were generally larger. In most cases, a large perforator with a superficial, straight intramuscular course could be identified a mean of $10 \mathrm{~cm}$ distal to the popliteal crease and an average of $2 \mathrm{~cm}$ from the posterior midline. Based on the above findings, we successfully used this flap in five clinical cases.

\section{Keywords}

- cadaveric

- anatomy

- sural artery perforator flap

- gastrocnemius muscle

Conclusion Perforators of the medial sural artery were arranged in a medial and a lateral row. Use of perforators from the lateral row, nearer the posterior midline, is preferable as these are usually larger in size. A consistent major perforator could always be identified in all specimens. With increased safety and confidence in flap harvesting, the medial sural artery perforator flap may find wider clinical applications.

Clinical question: Therapeutic

Level of Evidence: IV
\end{abstract}

\section{Introduction}

Taylor \& Daniel ${ }^{1}$ first suggested the medial sural vessels as the basis of a new flap donor site in 1975 and, two decades later, Montegut ${ }^{2}$ presented the first clinical cases of the medial sural artery perforator flap. Subsequently, Cavadas ${ }^{3}$ and other authors $^{4-11}$ reported small clinical series of its use and emphasized its many advantages and limited donor morbidity. In situations when a thin and pliable flap is needed, the radial forearm flap has traditionally been the choice flap. ${ }^{12}$ received

January 30, 2012

accepted after revision

March 21, 2012

published online

June 28, 2012
Copyright (c) 2012 by Thieme Medical Publishers, Inc., 333 Seventh Avenue, New York, NY 10001, USA. Tel: +1(212) 584-4662.
DOI http://dx.doi.org/ 10.1055/s-0032-1315778. ISSN 0743-684X. 
The medial sural artery perforator flap is comparable to the radial forearm flap in its thinness and pliability. It is therefore a suitable alternative for such defects with much lower donor site morbidity. The medial sural artery perforator flap is, however, more technically demanding, with a need for intramuscular dissection to raise the flap. Compounded by the lack of clearly described surgical anatomy of the flap, many surgeons have avoided utilizing this flap. Previous anatomic studies have sought to identify the locations of the medial sural artery perforators. Although their approximate vertical distances from the popliteal crease have been described, it remains difficult to predict its location preoperatively. This is particularly important when a small flap is needed, as the flap needs to be centered exactly over the dominant perforator. This study aimed to further clarify the vascular anatomy of this flap and identify the most consistent major perforator to facilitate safer flap harvesting.

\section{Methods and Materials}

Ten lower limbs from fresh adult cadavers were injected with red latex. The limbs were positioned prone with the knee extended, and subfascial dissection was begun from the posterior midline and advanced medially. The number of major (subfascial diameter $>1.0 \mathrm{~mm}$ ) and minor perforators were noted. The vertical distance from the popliteal crease and the horizontal distance from the posterior midline of the leg were recorded in centimeters. Each sizable perforator was followed by intramuscular dissection to its origin, and the pedicle length was recorded.

\section{Results}

An average of 4.4 perforators (range $=3$ to 8 ) was found to pierce the medial gastrocnemius muscle in each lower limb. At least one major perforator was identified in all specimens $($ mean $=2)$. Eight of ten legs had two or more sizable perforators.

The medial sural artery originated from the popliteal artery. After running for a distance of 3 to $6 \mathrm{~cm}$, the medial sural artery typically divided into two branches (a medial and lateral branch) within the substance of the medial gastrocnemius muscle. In one specimen, the medial sural artery did not give off any divisions and in another it was observed to divide into three branches. Perforators were given off at intervals along the course of each branch, and these musculocutaneous perforators supplied the skin over the medial calf. We noted that perforators of the medial sural artery were consistently arranged in two vertical rows (a medial and a lateral row) along the axis of the leg. By dividing the medial gastrocnemius bulk into equal thirds vertically, the lines between adjacent thirds serve as approximate surface landmarks for the medial and lateral rows of perforators (-Fig. 1). Perforators were more plentiful and larger in the lateral row, which was situated closer to the posterior midline of the leg, $\sim 2 \mathrm{~cm}$ away (-Fig. 2). Large perforators were identified in the medial row ( $\sim 6 \mathrm{~cm}$ medial to the posterior midline) in only five of the ten lower limbs.

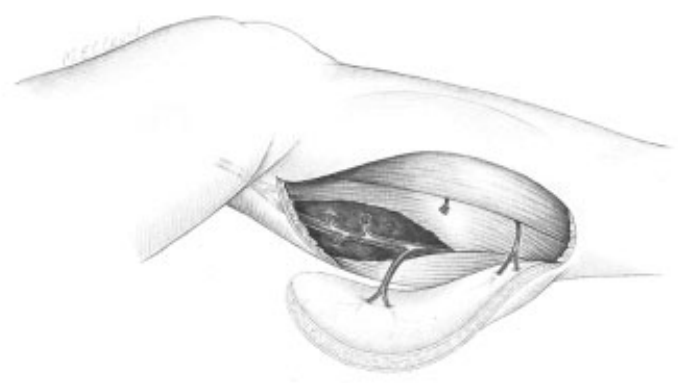

Figure 1 The medial sural artery divides into two branches and perforators are given off along each of their lengths. The perforators are orientated in two parallel rows, which can be landmarked by roughly dividing the gastrocnemius muscle bulk into thirds.

In nine out of ten specimens, a consistent large perforator could be identified between $10 \mathrm{~cm}( \pm 2 \mathrm{~cm})$ from the popliteal crease and an average $2 \mathrm{~cm}( \pm 0.5 \mathrm{~cm})$ from the posterior midline. This reliable perforator had a superficial axial intramuscular course when traced to its origin from the lateral branch of the medial sural artery (-Fig. 3). In one specimen, this perforator was located more distally, $15 \mathrm{~cm}$ from the popliteal crease. A second perforator was found in the medial row at $10 \mathrm{~cm}$ distal to the popliteal crease.

Most perforators appeared to arise in the proximal half of the medial gastrocnemius muscle bulk; although in half the leg specimens, at least one other reliable perforator could be identified $16 \mathrm{~cm}( \pm 2 \mathrm{~cm})$ from the popliteal crease (-Fig. 4). Pedicle length of 11 to $19 \mathrm{~cm}$ (mean $=13.7 \mathrm{~cm}$ ) could be obtained at completion of flap harvest. The mean diameter of the major perforators at the subfascial level ranged from 1 to $2 \mathrm{~mm}$ (mean $=1.5 \mathrm{~mm}$ ). The medial sural artery itself averaged 2.5 to $3 \mathrm{~mm}$ in diameter.

Based on this information, we modified our surgical approach to harvesting the medial sural artery perforator flap. Preoperatively, the medial calf was examined with the handheld Doppler. The preferred perforator was the one

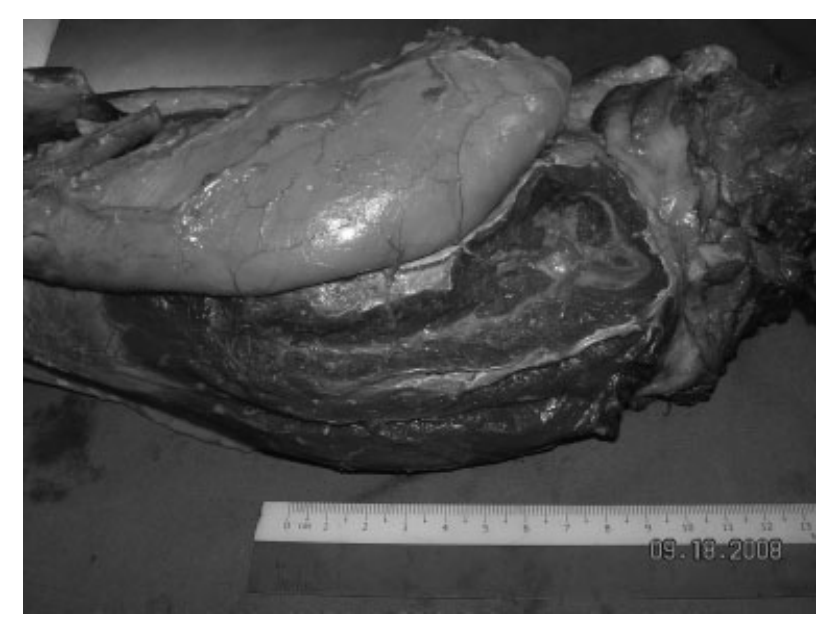

Figure 2 They are clustered $\sim 2 \mathrm{~cm}$ (lateral row) and $6 \mathrm{~cm}$ from the posterior midline (medial row). The proximal perforators are clustered an average of $10 \mathrm{~cm}$ from the popliteal crease, whereas the distal group of perforators lie an average of $15 \mathrm{~cm}$ from the popliteal crease. 


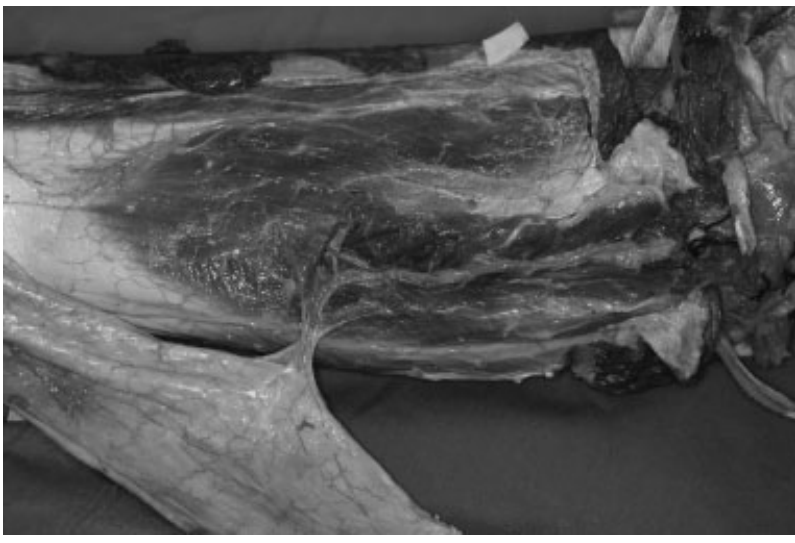

Figure 3 In nine of ten specimens, a consistent major perforator could be identified between $10 \mathrm{~cm}(+/-2 \mathrm{~cm})$ from the popliteal crease and an average $2 \mathrm{~cm}(+\mid-0.5 \mathrm{~cm})$ from the posterior midline. This perforator was large and had a superficial intramuscular course, facilitating intramuscular dissection.

located $\sim 10 \mathrm{~cm}$ from the popliteal crease and $2 \mathrm{~cm}$ from the midline. The flap was harvested with no tourniquet applied to enable observation of pulsation of the perforator prior to committing to its use as the vessel nourishing the flap. The lateral (nearer to the posterior midline) incision was made first, as this enabled the surgeon to get to the medial row where the location of the dominant perforator was most likely located. Having identified the perforator, the skin was completely islanded and intramuscular dissection completed to elevate the flap. The pedicle was mobilized to the origin of the medial sural artery from the popliteal artery, usually located at the level of the popliteal crease. Using this approach, we successfully used this flap in five clinical cases. The mean pedicle length was $12 \mathrm{~cm}$ (range $=9$ to $15 \mathrm{~cm}$ ). All flaps were successfully raised and survived uneventfully. In three cases, the donor site required skin grafting. In two cases where a small flap was harvested, the donor site could be closed primarily.

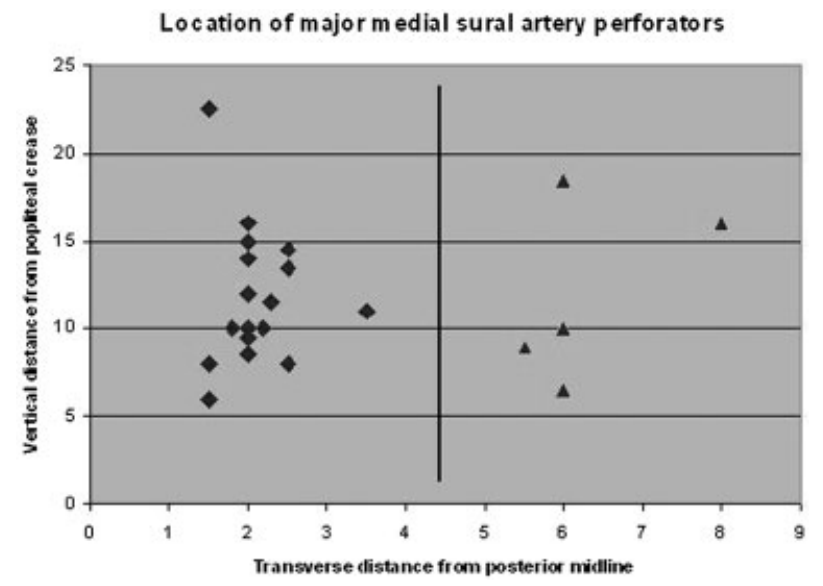

Figure 4 Distribution of major perforators ( $>1.0 \mathrm{~mm}$ ) Perforators in the lateral row (nearer the posterior midline of the leg) are more dominant.

\section{Case 1}

A 19-year-old female presented with a right dorsal hand defect after debridement of an abscess secondary to extravasation injury (-Fig. $\mathbf{5 a}$ ). The $3 \mathrm{~cm}$ by $5 \mathrm{~cm}$ defect with exposed extensor tendons was covered with a contralateral medial sural artery perforator flap. Preoperative examination with the handheld Doppler showed that the perforators were located in two rows, as expected from our cadaveric study (-Fig. 5b). A posterior midline incision was made first, and dissection proceeded subfascially. Intraoperatively, the medial sural artery divided into two branches $2 \mathrm{~cm}$ from its origin from the popliteal artery. Correspondingly, one perforator could be identified in the medial row $(10 \mathrm{~cm}$ from popliteal crease and $5 \mathrm{~cm}$ from the posterior midline), arising from the medial branch. Another more substantial perforator $(10 \mathrm{~cm}$ from popliteal crease and $2 \mathrm{~cm}$ from the posterior midline) arose from the lateral branch of the medial sural artery. Both perforators were completely unroofed, and the flap was islanded and raised based on both branches of the medial sural artery (-Fig. 5c). End-to-end anastomoses were performed between the medial sural artery and one vena comitans to the princeps pollicis artery and a dorsal cutaneous vein respectively. One side of the flap was left unsutured due to intraoperative swelling, and secondary suture was completed 4 days later. The donor site was skin-grafted. Healing was uneventful. Secondary debulking of the flap was performed with good results ( $\mathbf{- F i g . ~ 5 d , ~ 5 e ) . ~}$

\section{Case 2}

A 22-year-old male smoker presented with a left foot degloving injury exposing the first metartarsophalangeal joint. The defect measured $7 \mathrm{~cm}$ by $6 \mathrm{~cm}$ (-Fig. 6a). The patient was positioned prone, and dissection proceeded from a posterior midline incision. An ipsilateral medial sural artery perforator flap was harvested based on a large lateral row perforator $(8 \mathrm{~cm}$ from popliteal crease and $2 \mathrm{~cm}$ medial to posterior midline) arising from the lateral branch of medial sural artery (-Fig. 6b). The patient was turned back supine and end-toend anastomoses were performed between the medial sural artery pedicle to the dorsalis pedis artery, one accompanying vena comitans and a branch of the great saphenous vein. The donor site was covered with an unmeshed split-thickness skin graft (-Fig. 6c). Secondary flap debulking was performed 6 months later with good final contour of the flap (-Fig. 6d, 6e).

\section{Discussion}

Since its description in 2001 by Hallock $^{13}$ and, independently, by Cavadas, ${ }^{3}$ the medial sural artery perforator flap has been shown to be a promising flap for coverage of regional and distant defects. It is muscle sparing with limited donor morbidity; because of intramuscular dissection, its pedicle length and arc of rotation are superior to the conventional medial gastrocnemius myocutaneous flap. ${ }^{5}$ It has found applications as a pedicled flap for defects of the knee and upper third of the lower leg ${ }^{4,5}$ and as a free flap for head and 

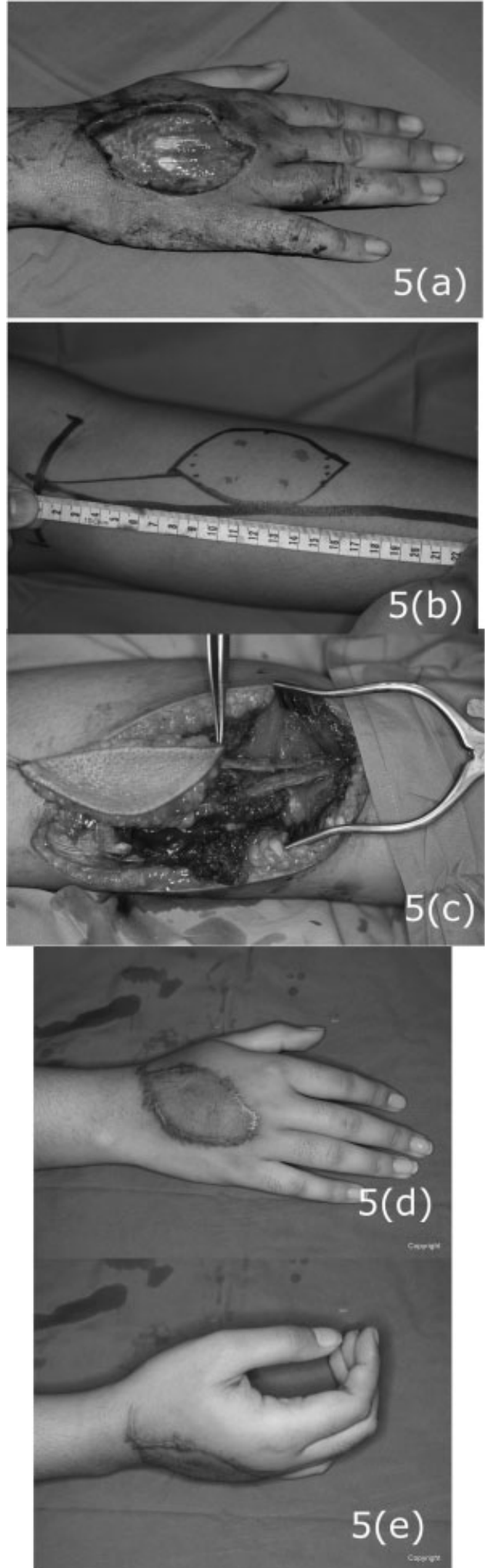

Figure 5 (a) A 19-year-old female presented with a right dorsal hand defect with exposed extensor tendons. (b) A left medial sural artery perforator flap was designed and the perforators were localized preoperatively. (c) The flap was harvested based on two perforators, one arising from each branch of the medial sural artery. The more substantial perforator arose from the lateral branch of the medial sural artery. (d, e) Final result shows acceptable contour and excellent hand function

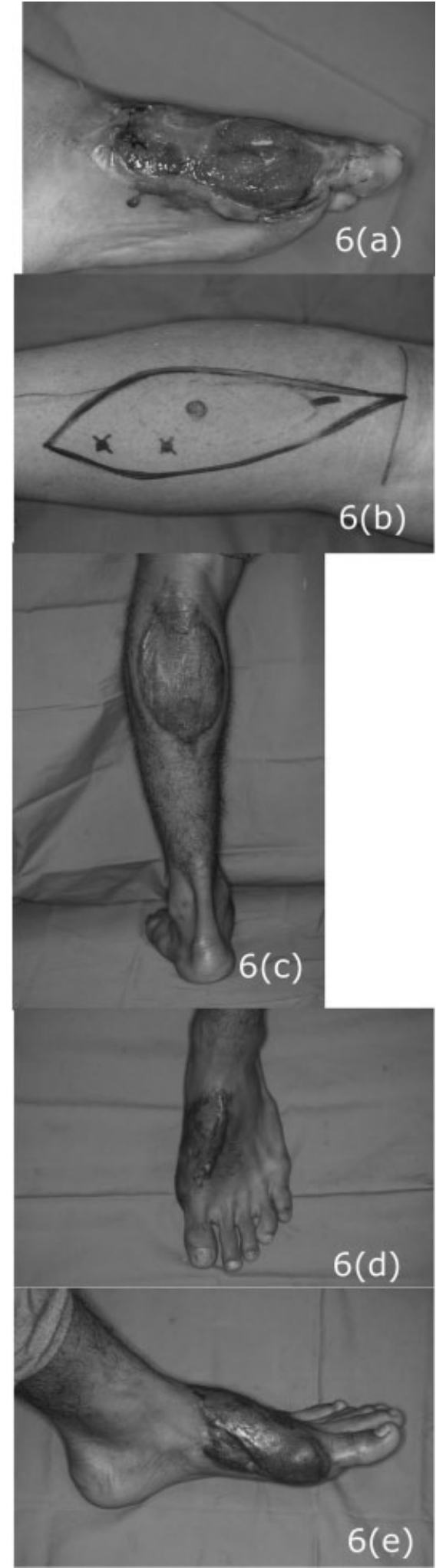

Figure 6 (a) A 22-year-old male presented with a left foot degloving injury with exposure of the first metatarsophalangeal joint. (b) An ipsilateral medial sural artery perforator flap was harvested based on a large lateral row perforator $(8 \mathrm{~cm}$ from popliteal crease and $2 \mathrm{~cm}$ medial to posterior midline) arising from the lateral branch of the medial sural artery. (c) Appearance of the donor site after skingrafting. (d, e) Good final contour of the flap after secondary debulking. 
neck and limb reconstruction. ${ }^{6-11,14}$ Inclusion of the plantaris tendon, sural nerve, and gastrocnemius muscle increases its versatility as a chimeric flap. ${ }^{15,16}$

Despite its advantages, concerns limiting its use include the variability of its vascular anatomy and the need for intramuscular dissection. ${ }^{17}$ Cavadas $^{3}$ first reported that the perforating vessels were clustered 8.5 to $19 \mathrm{~cm}$ from the popliteal crease. In the most frequent case when two perforators were present, Thione et al ${ }^{18}$ concluded that the proximal one was at an average distance of $10.7 \mathrm{~cm}$ (range $=8$ to $13 \mathrm{~cm}$ ) from the popliteal crease, and the distant one was at an average distance of $16.3 \mathrm{~cm}$ (range $=12.5$ to $18 \mathrm{~cm}$ ). In comparison, studies on Asian populations postulated that the perforators were located 1 to $2 \mathrm{~cm}$ more proximally due to either shorter leg length ${ }^{19}$ or skin folding with flap harvest in knee flexion. ${ }^{20}$

Consistent with previous studies, ${ }^{3,18-21}$ we found the proximal and distal perforators to be located at an average of $10 \mathrm{~cm}$ and $16 \mathrm{~cm}$ from the popliteal crease. Although the vertical distances are quite well described, their locations in the horizontal plane with respect to the posterior midline remains conflicting and poorly defined. ${ }^{20,22}$ We found that the medial sural artery divides into two branches ${ }^{14}$ shortly after it pierces the medial gastrocnemius muscle: a medial and a lateral branch. These two branches traverse vertically down the muscle and give off musculocutaneous perforators, which are thus arranged in two parallel rows $\sim 2 \mathrm{~cm}$ and $6 \mathrm{~cm}$, respectively, from the posterior midline of the leg. The lateral row perforators are commonly larger and more dominant. In none of the specimens was the medial system dominant. In all except one leg, the most consistent major perforator was found $\sim 10 \mathrm{~cm}$ from the popliteal crease and $2 \mathrm{~cm}$ from the posterior midline. This reliable perforator had a superficial straight intramuscular course, just beneath the surface of the muscle, thus facilitating intramuscular dissection. Computed tomography (CT) angiography may be useful in confirming the presence of this perforator ${ }^{23}$; however, it may not always be available. Therefore, to successfully identify and preserve this largest perforator early on in the dissection, we propose that the initial incision when raising the flap should start from the posterior midline. Once it is located, the surgeon must visually confirm that the perforator is pulsating well. Sometimes the supposed "perforator" may in fact be a cutaneous nerve that does not carry an artery. The flap can then be completely islanded and the intramuscular dissection completed in the usual manner using perforator flap techniques. $^{24}$

When a second perforator is found in the distal half of the medial gastrocnemius, this is typically smaller than the proximal perforator but may still be chosen to provide an increased arc of flap rotation in the appropriate clinical situation.

\section{References}

1 Taylor GI, Daniel RK. The anatomy of several free flap donor sites. Plast Reconstr Surg 1975;56:243-253
2 Montegut WJ, Allen RJ. Sural artery perforator flap as an alternative for the gastrocnemius myocutaneous flap. Plast Reconstr Surg 1996;89:5113

3 Cavadas PC, Sanz-Giménez-Rico JR, Gutierrez-de la Cámara A, Navarro-Monzonís A, Soler-Nomdedeu S, Martínez-Soriano F. The medial sural artery perforator free flap. Plast Reconstr Surg 2001;108:1609-1615, discussion 1616-1617

4 Umemoto Y, Adachi Y, Ebisawa K. The sural artery perforator flap for coverage of defects of the knee and tibia. Scand J Plast Reconstr Surg Hand Surg 2005;39:209-212

5 Shim JS, Kim HH. A novel reconstruction technique for the knee and upper one third of lower leg. J Plast Reconstr Aesthet Surg 2006;59:919-926, discussion 927

6 Kim ES, Hwang JH, Kim KS, Lee SY. Plantar reconstruction using the medial sural artery perforator free flap. Ann Plast Surg 2009;62: 679-684

7 Chen SL, Chuang CJ, Chou TD, Chen TM, Wang HJ. Free medial sural artery perforator flap for ankle and foot reconstruction. Ann Plast Surg 2005;54:39-43

8 Chen SL, Chen TM, Lee CH. Free medial sural artery perforator flap for resurfacing distal limb defects. J Trauma 2005;58:323-327 Erratum in: J Trauma 2005;58:763

9 Chen SL, Yu CC, Chang MC, Deng SC, Wu YS, Chen TM. Medial sural artery perforator flap for intraoral reconstruction following cancer ablation. Ann Plast Surg 2008;61:274-279

10 Chen SL, Chen TM, Dai NT, Hsia YJ, Lin YS. Medial sural artery perforator flap for tongue and floor of mouth reconstruction. Head Neck 2008;30:351-357

11 Xie RG, Gu JH, Gong YP, Tang JB. Medial sural artery perforator flap for repair of the hand. J Hand Surg Eur Vol 2007;32:512-517

12 Wong CH, Lin JY, Wei FC. The bottom-up approach to the suprafascial harvest of the radial forearm flap. Am J Surg 2008;196: e60-e64

13 Hallock GG. Anatomic basis of the gastrocnemius perforator-based flap. Ann Plast Surg 2001;47:517-522

14 Kao HK, Chang KP, Chen YA, Wei FC, Cheng MH. Anatomical basis and versatile application of the free medial sural artery perforator flap for head and neck reconstruction. Plast Reconstr Surg 2010;125:1135-1145

15 Sano K, Hallock GG, Hamazaki M, Daicyo Y. The perforator-based conjoint (chimeric) medial Sural (MEDIAL GASTROCNEMIUS) free flap. Ann Plast Surg 2004;53:588-592

16 Hallock GG. Chimeric gastrocnemius muscle and sural artery perforator local flap. Ann Plast Surg 2008;61:306-309

17 Hallock GG, Sano K. The medial gastrocnemius sural perforator free flap: an 'ideal' prone position skin flap. Ann Plast Surg 2004;52:184-187

18 Thione A, Valdatta L, Buoro M, Tuinder S, Mortarino C, Putz R. The medial sural artery perforators: anatomic basis for a surgical plan. Ann Plast Surg 2004;53:250-255

19 Okamoto H, Sekiya I, Mizutani J, Otsuka T. Anatomical basis of the medial sural artery perforator flap in Asians. Scand J Plast Reconstr Surg Hand Surg 2007;41:125-129

$20 \mathrm{Kim} \mathrm{HH}$, Jeong JH, Seul JH, Cho BC. New design and identification of the medial sural perforator flap: an anatomical study and its clinical applications. Plast Reconstr Surg 2006;117:1609-1618

21 Torres LR, Teixeira WGJ, Setani EO, Wei TH, Zumiotti A. Skin flap of medial gastrocnemius muscle's perforating arteries. An anatomical study. Acta Ortop Bras 2007; 15:40-42

22 Hallock GG. A primer of schematics to facilitate the design of the preferred muscle perforator flaps. Plast Reconstr Surg 2009;123: 1107-1115

23 Higueras Suñé MC, López Ojeda A, Narváez García JA, et al. Use of angioscanning in the surgical planning of perforator flaps in the lower extremities. J Plast Reconstr Aesthet Surg 2011;64: 1207-1213

24 Wong $\mathrm{CH}$, Wei FC. Anterolateral thigh flap. Head Neck 2010;32:529-540 Review 